Annales Geophysicae (2002) 20: 139-150 (c) European Geophysical Society 2002

\title{
Electromagnetic waves and bursty electron acceleration: implications from Freja
}

\author{
L. Andersson ${ }^{1,4}$, J.-E. Wahlund ${ }^{2}$, J. Clemmons ${ }^{3}$, B. Gustavsson ${ }^{1}$, and L. Eliasson ${ }^{1}$ \\ ${ }^{1}$ Swedish Institute of Space Physics, Kiruna, Sweden \\ ${ }^{2}$ Swedish Institute of Space Physics, Uppsala, Sweden \\ ${ }^{3}$ NASA Goddard Space Flight Center, Greenbelt, USA \\ ${ }^{4}$ Now at Laboratory of Atmospheric and Space Physics, University of Colorado, USA
}

Received: 16 November 2000 - Revised: 2 July 2001 - Accepted: 9 July 2001

\begin{abstract}
Dispersive Alfvén wave activity is identified in four dayside auroral oval events measured by the Freja satellite. The events are characterized by ion injection, bursty electron precipitation below about $1 \mathrm{keV}$, transverse ion heating and broadband extremely low frequency (ELF) emissions below the lower hybrid cutoff frequency (a few $\mathrm{kHz}$ ). Largescale density depletions/cavities, as determined by the Langmuir probe measurements, and strong electrostatic emissions are often observed simultaneously. A correlation study has been carried out between the $\boldsymbol{E}$ and $\boldsymbol{B}$ field fluctuations below $64 \mathrm{~Hz}$ and $10 \mathrm{~Hz}$, respectively, (the DC instruments upper threshold) and the characteristics of the precipitating electrons. This study revealed that the energisation of electrons is indeed related to the broadband ELF emissions and that the electrostatic component plays a predominant role during very active magnetospheric conditions. Furthermore, the effect of the ELF electromagnetic emissions on the larger scale field-aligned current systems has been investigated, and it is found that such an effect cannot be detected. Instead, the Alfvénic activity creates a local region of field-aligned currents. It is suggested that dispersive Alfvén waves set up these local field-aligned current regions and, in turn, trigger more electrostatic emissions during certain conditions. In these regions, ions are transversely heated, and large-scale density depletions/cavities may be created during especially active periods.
\end{abstract}

Key words. Ionosphere (particle acceleraton; wave-particle interactions) Magnetospheric physics (auroral phenomena)

\section{Introduction}

An Alfvén wave propagating from the magnetosphere towards the ionosphere will encounter a denser plasma and as a result, may change its characteristics. For instance, the Alfvén wave may be reflected (e.g. Haerendel, 1983; Knud-

Correspondence to: L. Andersson

(laila.andersson@lasp.colorado.edu) sen et al., 1990, 1992), dissipated (e.g. Clark and Seyler, 1999; Goertz, 1984), or broken down into other wave modes (e.g. Seyler et al., 1998). A dispersive Alfvén wave (DAW) (e.g. Lysak and Lotko, 1996; Stasiewicz et al., 2000, and references therein) may, therefore, enhance both its inertial and kinetic characteristics, and develop a field-aligned electric field in the wave front when it propagates at a small angle to the Earth's magnetic field. These Alfvén waves are, therefore, often suggested as candidates for auroral zone fieldaligned electron acceleration processes.

The low frequency part of the broadband extremely low frequency (ELF) emissions observed by spacecraft in the auroral zone is often considered to be Alfvénic in nature, with perpendicular wavelengths of the order of the electron inertial length $\left(\lambda_{e}\right)$ (e.g. Chmyrev et al., 1989; Louarn et al., 1994; Wahlund et al., 1994; Seyler et al., 1995), and to be associated with field-aligned electron bursts at suprathermal energies (Boehm et al., 1990; Knudsen and Wahlund, 1998; Wahlund et al., 1998; Chaston et al., 1999; Lynch et al., 1999; Ivchenko et al., 1999). This wave activity is also connected with transverse heating of ions (Knudsen and Wahlund, 1998; Knudsen et al., 1998) and might cause largescale density depletions.

The field-aligned currents moving into and out from the ionosphere are often described as being closed within the ionosphere. The conductivity in the ionosphere is in this scenario connected to the strength of the field-aligned current and, hence, the particle acceleration. When a widespread region of DAW activity exists, this scenario will change to a local region of very strongly fluctuating field-aligned currents of several $100 \mu \mathrm{A} / \mathrm{m}^{2}$ with a rather weak contribution to the overall large-scale current systems.

The Freja mission is dedicated to observing the space plasma environment in polar regions with an orbit altitude of 900-1700 km. In this paper, we apply the formalism of the concept of DAW (e.g. Lysak and Lotko, 1996) to the ELF electric and magnetic field emissions measured by the Freja spacecraft in order to show that these measurements are consistent with such a model. Other observed parameters, such 
as those associated with field-aligned electron bursts, transverse ion heating, field-aligned current systems and the appearance of large-scale plasma density depletions/cavities, are discussed in terms of a DAW model. In Sects. 1 and 2 the characteristics of four Freja passages through the dayside auroral region are presented and discussed. In Sect. 3, a detailed study of the ELF emissions below $64 \mathrm{~Hz}$ is presented and discussed in terms of a DAW model. In Sect. 4, the expected field-aligned electric fields and propagation angles are derived from the electric and magnetic field measurements under the assumption that they are produced by dispersive Alfvén waves. In addition, the DAW associated field-aligned currents are investigated with regard to the effect on fieldaligned current systems. A correlation study between the electric and magnetic ELF fields and field-aligned electron burst characteristics is described. The correlation with the DC electric field and DC magnetic field is made to 64 and $10 \mathrm{~Hz}$, respectively, due to the instruments thresholds. Finally, we make some concluding remarks regarding the overall interpretation of the observed processes in terms of waveparticle interactions caused by dispersive Alfvén waves.

\section{The Freja instrumentation and orbit characteristics}

The Swedish/German satellite Freja passes the northern auroral region at an altitude of about $1700 \mathrm{~km}$ and at an inclination of $63^{\circ}$. It is a sun-pointing spacecraft with a spin period of $6 \mathrm{~s}$. Detailed information about the Freja experiments can be found in a special Freja issue of Space Science Reviews (see Boehm et al., 1994; Eliasson et al., 1994; Holback at al., 1994; Marklund et al., 1994). Relevant experimental information for this study is discussed where necessary.

\section{General characteristics of the four Freja auroral oval crossings}

Data are presented from four Freja satellite passages through the dayside auroral region during ion injection events in Table 1. For convenience, these orbits are referred to as $I, I I, I I I$, and $I V$, respectively, corresponding to actual orbits 5200 (3 November 1993), 5265 (8 November 1993), 5292 (10 November 1993), and 6653 (21 February 1994). A short description of the general observational characteristics is given here, and more detailed information regarding these particular data can be found in Andersson et al. (2001).

Figure 1 displays the overview of particle and wave data from all four orbits, and Fig. 2 shows the corresponding energy densities, as well as the DC electric and magnetic field waveforms.

\subsection{Ion injection and heating}

All four orbits are characterized by clear ion injections with energies from $500 \mathrm{eV}$ up to the TICS instrument threshold of $4.3 \mathrm{keV}$ (Fig. 1, panel b). The downward proton energy flux
Table 1. Time, location and solar wind

\begin{tabular}{lrccc}
\hline & I & II & III & IV \\
\hline Orbit & 5200 & 5265 & 5292 & 6653 \\
Date & 931103 & 931108 & 931110 & 940221 \\
Start (UT) & 184630 & 164300 & 174700 & 170130 \\
End (UT) & 184900 & 165100 & 175200 & 170430 \\
\hline CGLAt $\left(^{\circ}\right)$ & 74 & 74 & 74 & 69 \\
Height $(\mathrm{km})$ & 1735 & 1675 & 1715 & 1565 \\
MLT(start) & 13 & 10 & 10 & 7 \\
MLT(end) & 14 & 13 & 12 & 8 \\
K $_{p}$ & 2 & 3 & 3 & $6-$ \\
SW $(\mathrm{km} / \mathrm{s})$ & 300 & 550 & 450 & 700 \\
IMF $\mathrm{B}_{z}(\mathrm{nT})$ & nan & $\sim-2$ & $\sim-2$ & $\sim+2$ \\
\hline & & & &
\end{tabular}

The values in the table are given for the middle point of the ion injection (if not else stated). For all orbits the satellite moved from dawn to dusk.

(Fig. 2, panel a) is calculated by integrating over all TICS energies from $50 \mathrm{eV}$ to $4.3 \mathrm{keV}$ and over pitch-angles between $0^{\circ}$ and $90^{\circ}$. The time resolution of this calculated ion energy flux is $400 \mathrm{~ms}$. Occasionally, these fluxes are somewhat spin modulated due to limitations in pitch-angle coverage. Downward ion energy fluxes of $0.01-1 \mathrm{~mW} / \mathrm{m}^{2}$ exist during the observed ion injections. The ion injections are accompanied by bursty electron precipitation, transverse ion heating as well as broadband ELF emissions up to the lower hybrid cutoff frequency (Fig. 1). Large-scale density depletions, as measured by the Langmuir probe, are only detected during the most active orbit, IV (Fig. 2, panel e). Intense transverse ion heating (primarily $\mathrm{O}^{+}$) is only encountered in orbit $I V$, for which it reaches energies of up to $\sim 400 \mathrm{eV}$, while the ion heating during the other three orbits reaches at most $\sim 50 \mathrm{eV}$. The spin modulated signature at low energies, primarily in the oxygen channel, is the RAM flux, as seen in Fig. 1, panel (a). On top of the RAM, the ion heating can be seen.

\subsection{Electron precipitation characteristics}

The electron bursts have downward energy fluxes (calculated from pitch-angles $0^{\circ}-90^{\circ}$ ) of the order of $0.01-10 \mathrm{~mW} / \mathrm{m}^{2}$ (Fig. 2, panels b and c), with the most intense events encountered in orbit $I V$. Panel (b) contains TESP data in the lower energy range of, $12-50 \mathrm{eV}$, which represents electrons moving with velocities below the local Alfvén velocity $\left(v_{A}\right)$. Panel (c) includes almost the full energy range of the TESP instrument $(36 \mathrm{eV}-25 \mathrm{keV})$. The time resolution of the electron measurements is $62.5 \mathrm{~ms}$.

The most field-aligned electron precipitation can also be found in orbit $I V$ (Fig. 2, panel (d); red line denotes $0^{\circ}$ pitchangle, and black denoted $90^{\circ}$ ). In all four orbits, the flux in $45^{\circ}$ (not shown) and the $90^{\circ}$ pitch-angle is equal, but during 
(1)

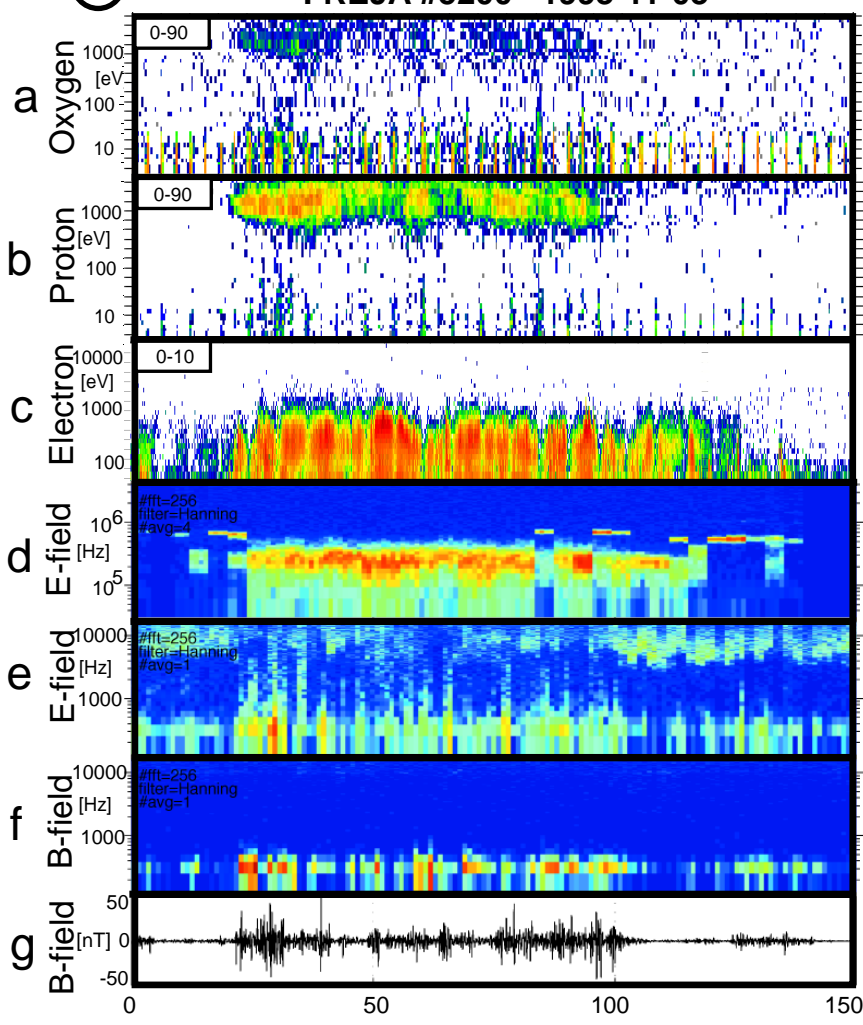

(III)

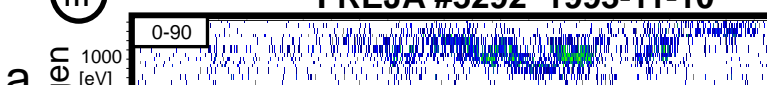

a

ญั่

b

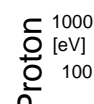

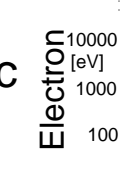

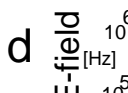

山่ $10^{5}$

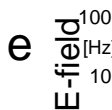

$f$

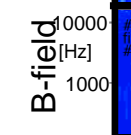

$g$

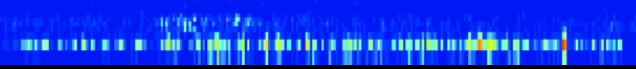

(11)

FREJA \#5265 1993-11-08
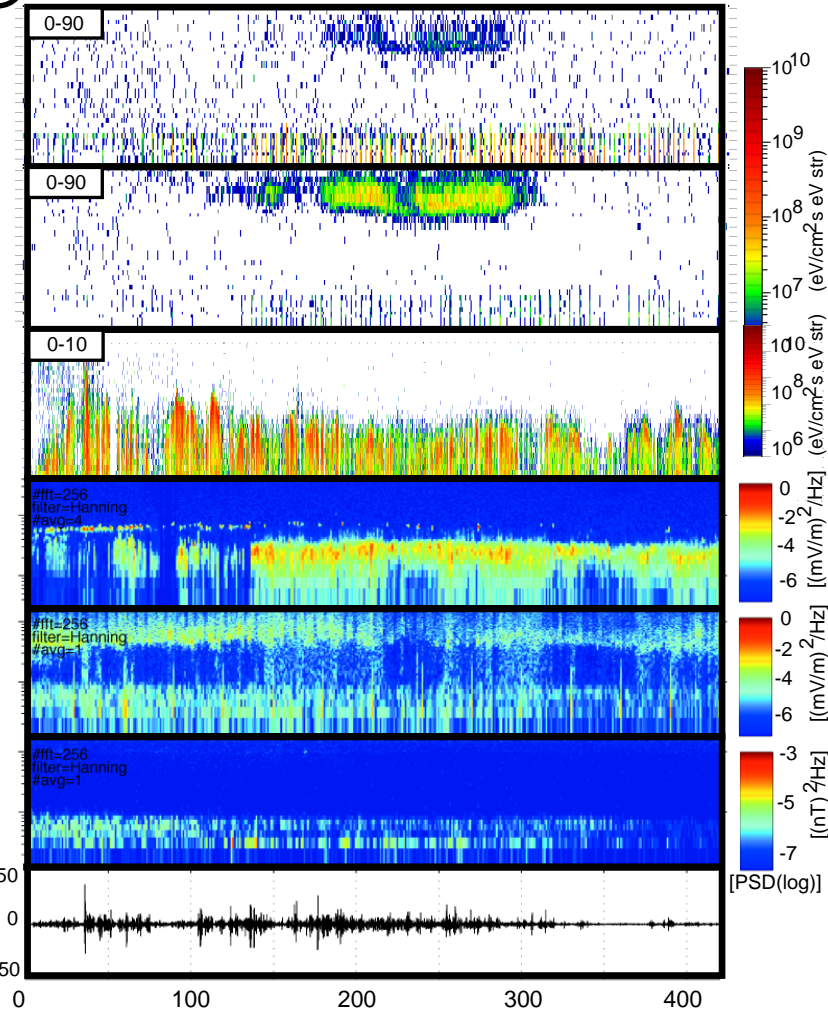

(IV)

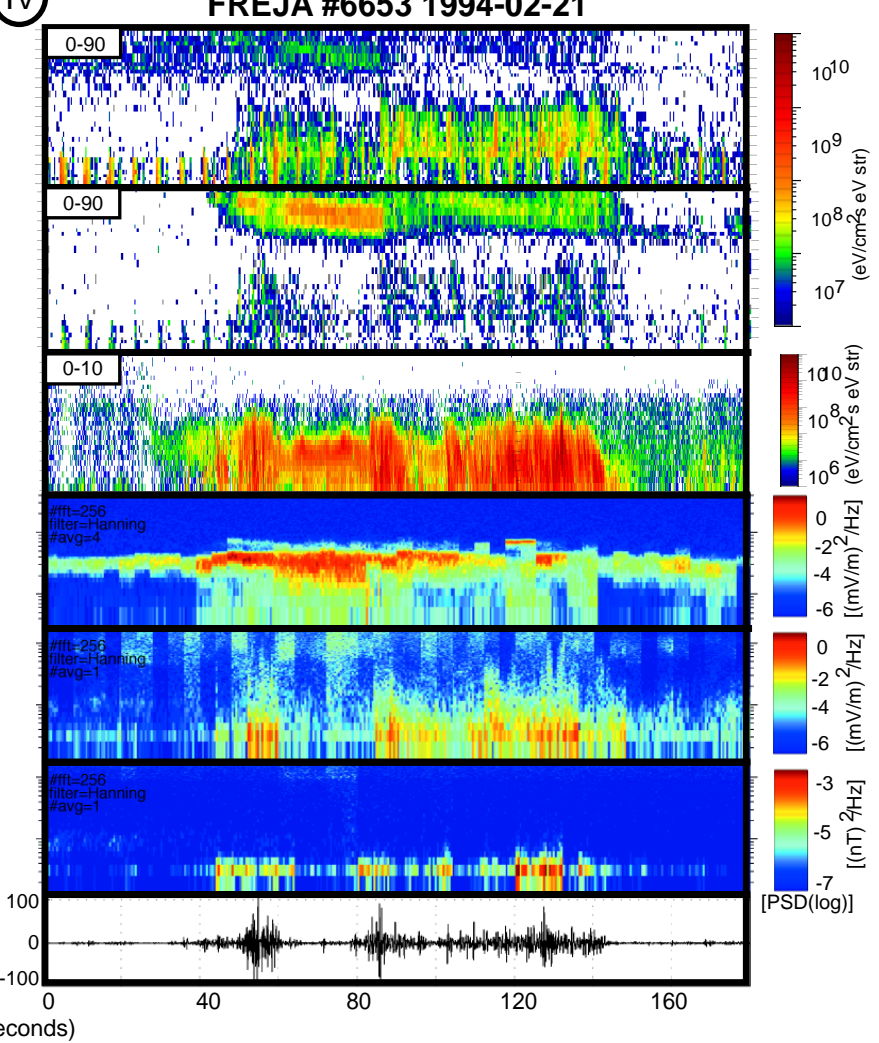

Fig. 1. Overview data from four Freja satellite passes of the northern dayside auroral region. The panels display from the top; $\mathrm{O}^{+}(\mathrm{TICS}), \mathrm{H}^{+}$ (TICS), field-aligned downward moving electrons (TESP), HF electric wave components (F4), MF electric and magnetic wave components (F4), and the AC magnetic field (F2), respectively. 

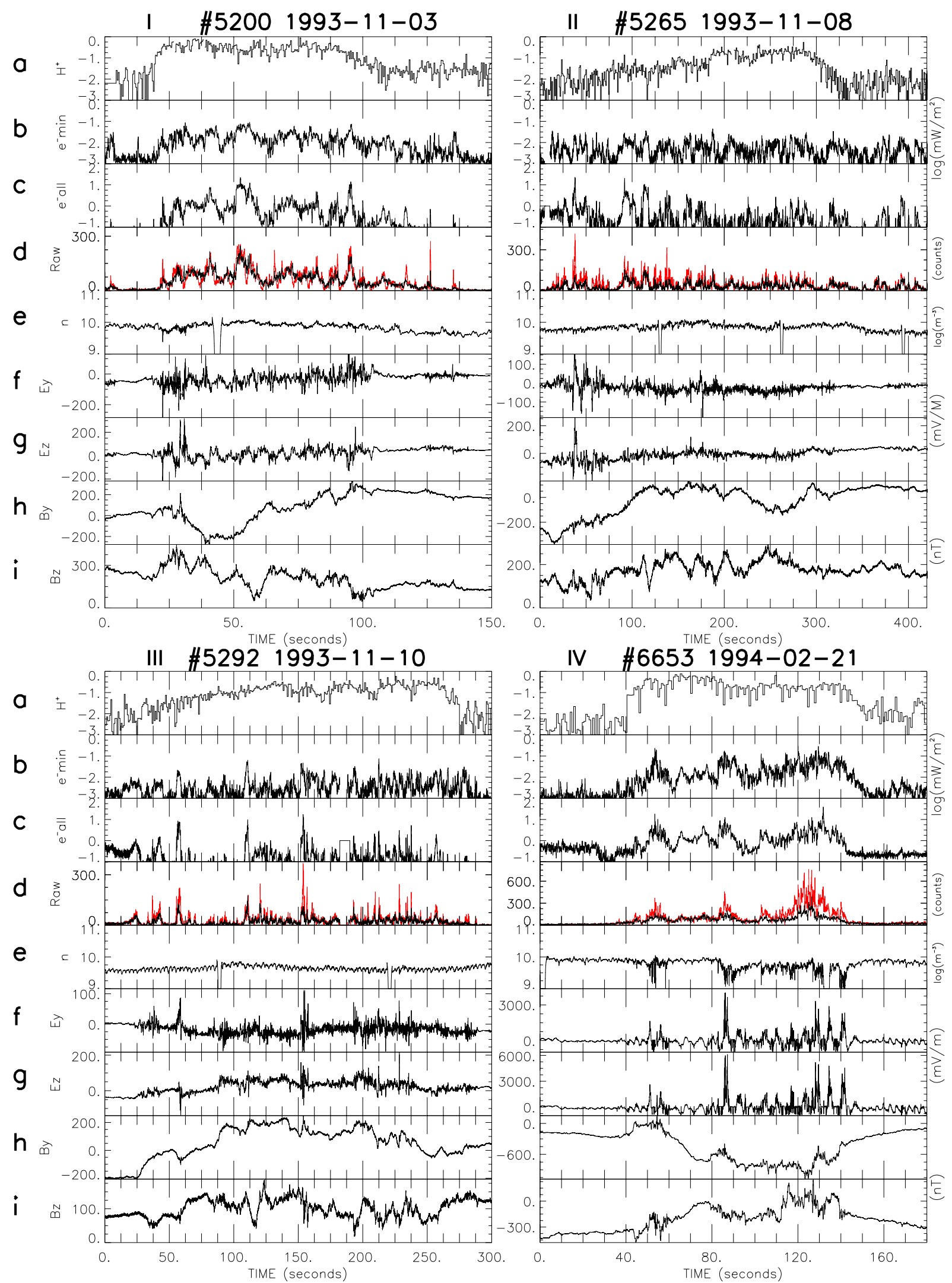

Fig. 2. Data from the same four Freja satellite passes as shown in Fig. 1. The panels show from the top; proton $(50 \mathrm{eV}-4.3 \mathrm{keV})$, electron $(12-50 \mathrm{eV})$ and electron $(36 \mathrm{eV}-25 \mathrm{keV})$ downward energy fluxes $\left(\mathrm{mW} / \mathrm{m}^{2}\right)$; the total electron counts for two different pitch-angles $\left(0^{\circ}\right.$ red and $90^{\circ}$ black); the Langmuir probe measured density; the DC electric and magnetic fields perpendicular to the model magnetic field lines. In panel (b) to (d), small data gaps can be found. 
the bursts of electrons, the $0^{\circ}$ pitch-angle fluxes are much higher. In orbit $I$ one sector which measures the field-aligned electron flux has a lower sensitivity; this leads to a spin modulated signal and the field-align burst is not clearly shown. In orbits $I I$ and $I I I$, bursts appear more sporadically, while in orbit $I V$, the electron bursts occur more frequently in time.

\subsection{Cold plasma characteristics}

A set of spherical Langmuir probes continuously samples the current from the plasma at a fixed positive bias voltage. This current is proportional to $n_{e} * \sqrt{T_{e}}$, where $n_{e}$ and $T_{e}$ are the electron density and temperature. Usually the variation in the electron temperature is small and slow, and the Langmuir probe current variation is assumed to be solely due to plasma density variations. When possible, we have used the narrow band HF Langmuir emissions near the plasma frequency, $\omega_{p e}$, as well as the intermittent Langmuir probe bias voltage sweeps to confirm that the sampled probe current is primarily due to the variations in $n_{e}$, even though a variation in $T_{e}$ may account for a current decrease by up to a factor of 2 during active periods.

The regularly measured probe sweeps are seen as dropouts in Fig. 2 (panel e). In Table 2, measurements from the analyzed probe sweeps, such as the plasma temperatures $\left(T_{i}\right.$, $\left.T_{e}\right)$ and the temperature ratios $\left(T_{i} / T_{e}\right)$, are presented together with some useful calculated plasma characteristics. As mentioned above, the ion heating is weak during orbits $I I$ and $I I I$, somewhat stronger in orbit $I$ and intense in orbit $I V$. It should be noted that it is the whole bulk ion population that is heated, not just the energetic tail of the ion distribution.

During orbit $I V$, several density drops (Fig. 2, panel e) are observed simultaneously with ion injection (panel a) and high electron count rates in the field-aligned direction, as compared to count rates at other pitch-angles (panel d). The four largest density drops (hereafter referred to as cavities) are centered around $+53,+88,+130$ and $+141 \mathrm{~s}$ into the plot. The highest electron count rates (120-130 s) have their main contribution from the lowest energies below $50 \mathrm{eV}$.

\subsection{Electromagnetic fields and wave characteristics}

It is clearly seen from Fig. 1 (AC-measurements, panels e and $\mathrm{f}$ ) that the broadband ELF emissions can reach frequencies above $1 \mathrm{kHz}$ and in some cases up to $6 \mathrm{kHz}$, which is close to the lower hybrid cutoff. It can also be seen that the emissions become increasingly electrostatic towards higher frequencies, while the magnetic component becomes dominant at the lowest frequencies (see panels $\mathrm{f}$ and $\mathrm{g}$, for $\mathrm{AC}$ magnetic field measurements). See Wahlund et al. (1998) for further details regarding the broadband ELF emissions. This study concentrates more on the lower frequency, and the more electromagnetic part of the ELF spectrum and its relationship to electron bursts, dayside ion injections and density depletions.

The DC electric and magnetic field instruments were operated somewhat differently during the four selected or- bits. The DC electric field measurements normally make use of two orthogonally positioned spherical probe pairs on $10.5 \mathrm{~m}$ long wire booms in the spin plane (i.e. $21 \mathrm{~m}$ between spheres). However, during orbit $I V$, one of the probe pairs was operated in a current collection mode allowing for $\delta n / n$ interferometry measurements, and as a consequence, only one probe pair measures the DC electric field on this orbit. This explains why the electric field on orbit $I V$ (Fig. 2, DCmeasurements, panels $\mathrm{f}$ and $\mathrm{g}$ ) is spin modulated. The sampling rates for orbits $I, I I$ and III are 768 samples/s for the DC electric field, and 128 samples/s for the DC magnetic field. On orbit $I V$, the DC electric field was sampled at 1536 samples/s and the DC magnetic field at 256 samples/s.

The DC electric and magnetic field measurements have been de-spun and are presented in a coordinate system for which the $x$-axis is along a model magnetic field line, positive $y$ points towards east, and positive $z$ points towards the equator. The Freja satellite measures the electric field in the spin plane of the spacecraft and, therefore, only gives a 2dimensional estimate of the total electric field. Therefore, we assume that the magnetic field-aligned component $\left(E_{x}\right)$ is zero. The assumption should be good since the field-aligned electric field component of a dispersive Alfvén wave is theoretically much smaller than the perpendicular electric field component.

The electric field fluctuations (Fig. 2, panels $f$ to $g$ ) are of extremely large amplitude during orbit $I V$. When the downward ion energy flux is close to $1 \mathrm{~mW} / \mathrm{m}^{2}$ the magnetic field data show a large gradient, and the electron distribution is isotropic with a loss cone. There is no density gradient, and the DC electric field shows just a spin modulation of about zero. On orbit II I, downward bursts of electrons are correlated with sharp changes in the magnetic field.

\section{Detailed study of the ELF emissions below $64 \mathrm{~Hz}$}

The time series of the DC electric and magnetic field measurements were analyzed by an overlapping sliding window Fast Fourier Transform (FFT) analysis (Fig. 3, panels a to e). In order to cover all frequencies below $64 \mathrm{~Hz}$, four different time windows were selected $(15,6,1$, and $0.3 \mathrm{~s}$ corresponding to the lowest non-DC frequencies of $0.133,0.333,2$ and $7.1 \mathrm{~Hz}$, respectively). Each time window was weighted with a Hamming filter. The longer time windows are then used at the lower frequencies, while the shorter time windows are used at the higher frequencies in Fig. 3 (panels a to e). The wave spectrograms presented in Fig. 3 have the same apparent time resolution as the electron data (TESP, $62.5 \mathrm{~ms}$ ). This analysis corresponds very much to a wavelet analysis, although it is FFT-based.

\subsection{Poynting flux}

The wave energy per unit of time and area is represented by the spectral Poynting flux $\left(\boldsymbol{S}(f)=1 / \mu_{0} \boldsymbol{E}(f) \times \boldsymbol{B}(f)\right)$ in Fig. 3 (panel f). The Poynting flux along the Earth's magnetic 
Table 2. Values from the probe sweeps. Data in the top five rows originate from the evaluation of the probe sweeps. The values in the middle four rows are calculated using the assumption of a 100\% oxygen plasma. The last row shows the time of the probe sweeps.

\begin{tabular}{lrccccccc}
\hline ORBIT & $I$ & \multicolumn{1}{c}{$I I$} & $I I$ & $I I$ & $I I I$ & $I I I$ & $I V$ & $I V$ \\
\hline$N_{O^{+}} / N_{i, t o t}$ & 0.85 & 0.95 & 0.95 & 1.0 & 0.8 & 0.9 & 0.9 & 0.95 \\
$T_{e}[\mathrm{eV}]$ & 0.8 & 0.7 & 0.7 & 0.6 & 0.5 & 0.6 & 0.5 & 2.5 \\
$T_{i}[\mathrm{eV}]$ & 0.5 & 0.1 & 0.1 & 0.2 & 0.1 & 0.1 & 0.1 & 15 \\
$N_{e}\left[\mathrm{~cm}^{-3}\right]$ & 6100 & 5000 & 5000 & 4200 & 3000 & 2500 & 5000 & 4000 \\
$T_{i} / T_{e}$ & 0.63 & 0.14 & 0.14 & 0.33 & 0.2 & 0.17 & 0.2 & 6 \\
\hline$\sim B_{0}[\mathrm{nT}]$ & 29400 & 29900 & 29700 & 29500 & 29500 & 29400 & 30600 & 30800 \\
$\rho_{O^{+}}[\mathrm{m}]$ & 9.8 & 4.3 & 4.4 & 6.2 & 4.4 & 4.4 & 4.2 & 51.4 \\
$\lambda_{e}[\mathrm{~m}]$ & 68 & 75 & 75 & 82 & 97 & 106 & 75 & 84 \\
$v_{A}\left[10^{6} \mathrm{~m} / \mathrm{s}\right]$ & 2.1 & 2.3 & 2.3 & 2.5 & 2.9 & 3.2 & 2.4 & 2.7 \\
\hline $\mathrm{UT}$ & $18: 47: 10$ & $16: 45: 05$ & $16: 47: 25$ & $16: 49: 45$ & $17: 49: 00$ & $17: 51: 50$ & $17: 01: 30$ & $17: 03: 40$ \\
\hline
\end{tabular}

field is calculated from the wave data represented in panels (a) through (e) by

$S_{x}=R_{E}\left[\frac{1}{\mu_{0}}\left(E_{y} \cdot B_{z}-E_{z} \cdot B_{y}\right)\right]$

for each frequency component. It should be noted that the direction and magnitude of the total Poynting flux when integrated over frequency will depend on the frequency window used and their significance is unclear when treating broadband plasma wave emissions with several possible nonlinear wave modes involved. We do not present the direction of the spectral Poynting flux, only the magnitude $\left|S_{x}\right|$, since it shows very complex, small-scale variations, more suitable for detailed event studies on the time scale of seconds and is, therefore, left for a future paper. Here, we just note that the spectral Poynting flux is highest at the lowest frequencies, and correlates well with the downward electron energy flux (Fig. 2, panels b to d).

\subsection{The Alfvén velocity and the $\delta E_{\perp} / \delta B_{\perp}$-ratio}

Panels (g) and (h) in Fig. 3 display as functions of time the ratio between the electric and magnetic field fluctuations $\left(\delta E_{\perp} / \delta B_{\perp}\right)$ and the local Alfvén velocity $\left(v_{A}\right)$. The local Alfvén velocity $\left(v_{A}=B_{0} / \sqrt{\mu_{0} m_{i} n_{i}}\right)$ is calculated for four different ion compositions (using a $\mathrm{H}^{+}$and $\mathrm{O}^{+}$mixture with $100 \%, 88 \%, 47 \%, 0 \% \mathrm{H}^{+}$composition) and is based on the plasma density estimated from the Langmuir probe measurements.

At the lowest displayed frequencies, the ratio $\delta E_{\perp} / \delta B_{\perp}$ is lower than $v_{A}$ during the three first moderately active orbits $(I, I I$ and $I I I)$. This suggests that DC-like field-aligned current systems dominate the magnetic field fluctuations below about $0.5 \mathrm{~Hz}$. Between $0.5-7 \mathrm{~Hz}$, the $\delta E_{\perp} / \delta B_{\perp}$-ratio increases to a more stable plateau value somewhat above the local Alfvén velocity for a pure oxygen plasma. The drop at the highest displayed frequencies is probably an instrumental artifact; it might be that the magnetic emissions reach the noise level of the fluxgate magnetometer.

During orbit $I V$, the whole displayed frequency interval attains $\delta E_{\perp} / \delta B_{\perp}$-ratios in excess of the local Alfvén velocity for all possible $\mathrm{H}^{+}-\mathrm{O}^{+}$compositions. The ELF emissions are, therefore, more electrostatic than expected for a classical Alfvén wave, and inertial and/or kinetic effects are probably important. The large values at the lowest frequencies below $0.5 \mathrm{~Hz}$ may just be an effect of the fact that only one probe pair was used for electric field measurements.

\subsection{Dispersive Alfvén Waves (DAW)}

The term DAW is a general description covering inertial Alfvén waves (IAW, Goertz and Boswell, 1979) applicable in a plasma with $v_{t h, e}=\sqrt{k_{B} T_{e} / m_{e}}<v_{A}$ and $\beta=2 \mu_{0} n_{e} k_{B} T_{e} / B_{0}^{2}<m_{e} / m_{i}$, and kinetic Alfvén waves (KAW, Stefant, 1970; Hasegawa, 1976), applicable in a plasma with $v_{t h, e}>v_{A}$ and $\beta>m_{e} / m_{i}$. The concept originates from a development by Lysak and Lotko (1996), and is described in more detail in a review article by Stasiewicz et al. (2000). The dispersive properties of DAWs become important when the perpendicular wavelength becomes comparable to the ion gyroradius $\left(\rho_{i}\right)$ or the electron skin depth $\left(\lambda_{e}\right)$, i.e. when the quantities $k_{\perp} \rho_{i}$ and/or $k_{\perp} \lambda_{e}$ become comparable to or larger than 1. When this happens parallel electric fields develop which, in turn, can accelerate the electrons.

The dispersion relation for a low frequency DAW $\left(\omega<\omega_{c i}\right)$ propagating in a cold electron $\left(v_{t h, e}<v_{A}\right)$ low $\beta$ plasma can be described as

$\omega^{2}=k_{\|}^{2} v_{A}^{2} \frac{\left(1+k_{\perp}^{2} \rho_{i}^{2}\right)}{\left(1+k_{\perp}^{2} \lambda_{e}^{2}\right)}$

and the group velocity becomes

$\frac{\partial \omega}{\partial \boldsymbol{k}}=\hat{\boldsymbol{x}} \frac{v_{A}}{\left(1+k_{\perp}^{2} \lambda_{e}^{2}\right)^{1 / 2}}-\hat{\mathbf{y}} \omega \lambda_{e} \frac{k_{\perp} \lambda_{e}}{1+k_{\perp}^{2} \lambda_{e}^{2}}$. 


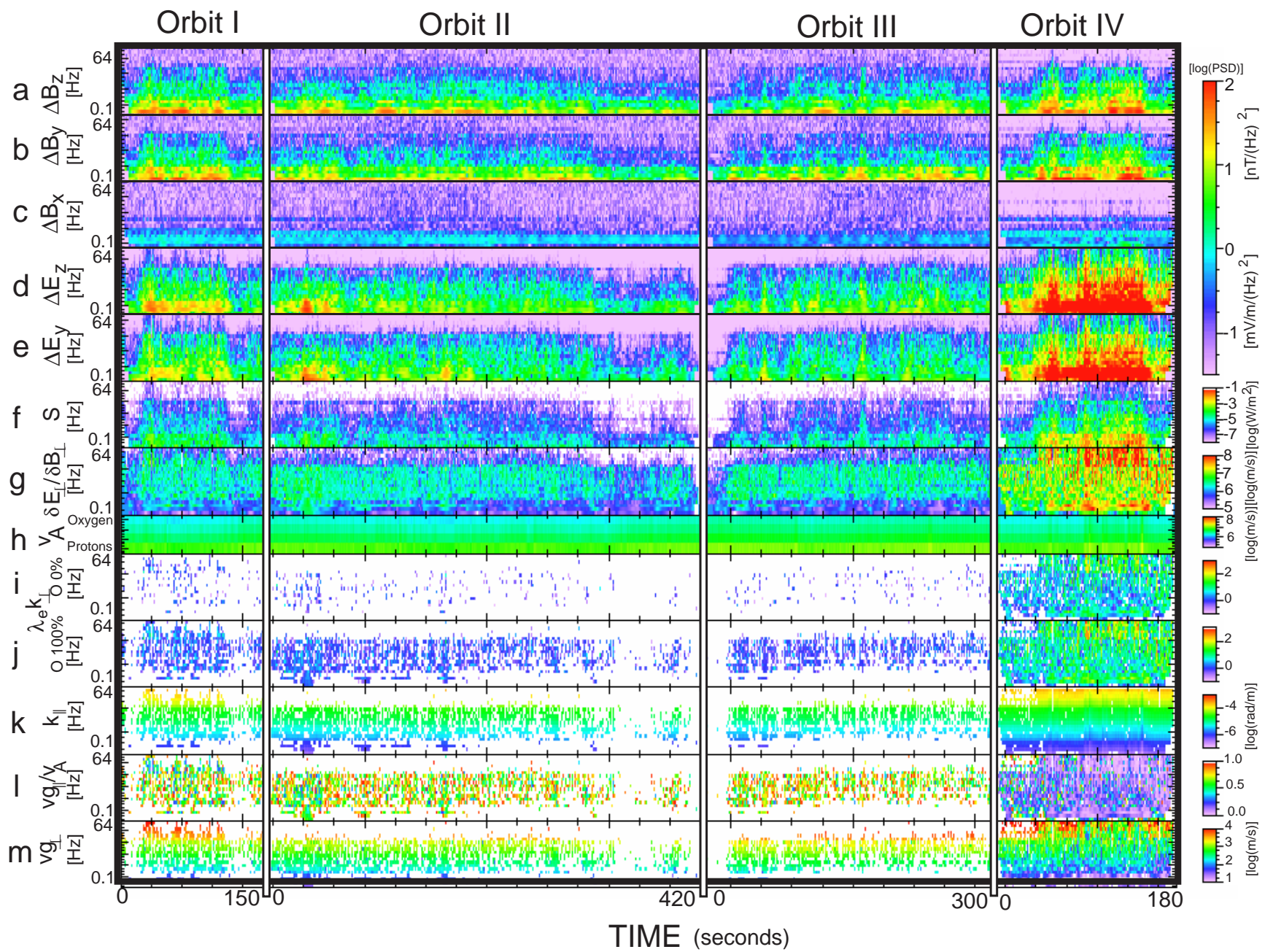

Fig. 3. More detailed ELF wave characteristics for the four orbits presented in Fig. 1. Panels from the top are, respectively; (a) to (e) the power spectral densities (PSDs) for the three magnetic field components and the two perpendicular electric field components; (f) the magnitude of the Poynting flux $(S)$; (g) $\delta E_{\perp} / \delta B_{\perp}$; (h) the Alfvén speed $\left(v_{A}\right)$ for four different plasma compositions; (i) to (j) the value of $\lambda_{e} k_{\perp}$ for a pure oxygen and a pure hydrogen plasma respectively; (k) the parallel wave vector $\left(k_{\|}\right)$; (l) the parallel group velocity of inertial Alfvén waves as a fraction of the local Alfvén speed; (m) the perpendicular group velocity of inertial Alfvén waves. See the text for more details.

Solving the dispersion relation in this cold electron limit gives a ratio of

$$
\left|\frac{E_{\perp}}{B_{\perp}}\right|=v_{A} \sqrt{\left(1+k_{\perp}^{2} \lambda_{e}^{2}\right)\left(1+k_{\perp}^{2} \rho_{i}^{2}\right)}
$$

and the relationship between the field-aligned and the perpendicular electric field becomes

$$
\frac{E_{\|}}{E_{\perp}}=\frac{k_{\|} k_{\perp} \lambda_{e}^{2}}{1+k_{\perp}^{2} \lambda_{e}^{2}} .
$$

These relationships do not take into account several possible nonlinear effects that can, for example, steepen the wavefront of a DAW, break the wave into higher frequency components, and enhance the parallel electric field further (e.g. Seyler et al., 1995; Seyler and Wahlund, 1996).

\subsection{Inertial term and $k_{\perp}$}

The perpendicular wave vector $\left(k_{\perp}\right)$ can be estimated from the Alfvén speed $\left(v_{A}\right)$ and the ratio $\delta E_{\perp} / \delta B_{\perp}$ (Eq. 4). From Table $2, \rho_{i}^{2} \ll \lambda_{e}^{2}$, which is a good approximation except possibly during the active periods on orbit $I V$. The value of $k_{\perp} \lambda_{e}$ is presented in Fig. 3 (panels $i$ and $j$ ) for pure hydrogen and oxygen plasmas, respectively. It should be noted that only points which satisfy $\delta E_{\perp} / \delta B_{\perp}>v_{A}$ for a pure oxygen plasma are kept in panels (i) through (m), and values below $v_{A}$ are white in those graphs (Eq. 4).

The value of $k_{\perp} \lambda_{e}$ increases significantly above zero when enhanced broadband electric field emissions appear (panels $\mathrm{d}$ and e), and values up to $k_{\perp} \lambda_{e} \sim 1-2$ can be detected during orbit $I V$. This corresponds to perpendicular wavelengths of around $250-500 \mathrm{~m}$ in the case of orbit $I V$ and values in excess of that for the other three orbits. 
The maximum possible Doppler shift (broadening) of the perpendicular wave vector (wave fields) due to the motion of the satellite is estimated to be $k_{\perp} v_{s c} \sim 10-20 \mathrm{~Hz}$ for orbits $I, I I$ and $I I I$ and $100-200 \mathrm{~Hz}$ for orbit $I V$. Thus, the enhanced ELF wave emissions which occur during orbit $I V$ may be heavily Doppler broadened (shifted) depending on the exact $\boldsymbol{k}$-vector distribution.

\subsection{Group velocity and $k_{\|}$}

From the knowledge of $k_{\perp}$, several other characteristics can be estimated: among them, the perpendicular and the parallel group velocities (panels 1 and $\mathrm{m}$ ), as well as the parallel wave vector (panel $\mathrm{k}$ ). These parameters are estimated assuming a pure oxygen plasma and by using $k_{\perp}$ calculated from panel (j). While $k_{\perp}$ is independent of frequency, except for frequencies above about $40 \mathrm{~Hz}$ during active parts of orbit $I V, k_{\|}$will depend on the frequency throughout the DAW dispersion relation. The parallel wavelengths estimated in this way are in the range of $100-1000 \mathrm{~km}$.

The parallel group velocity is close to the Alfvén speed during orbits $I, I I$ and $I I I$, while during orbit $I V$, the parallel group velocity becomes a fraction of the Alfvén speed, which is simply a consequence of the dispersion relation and the larger inertial terms. The perpendicular group velocity remains most of the time below $1 \mathrm{~km} / \mathrm{s}$, at least at the lower frequencies.

\section{Parallel electric fields}

The parallel electric field associated with a DAW can be calculated in various ways. We shall adopt three methods and compare the results; one method makes use of the magnetic field measurements, and the other two are calculated based on the perpendicular electric field and the above estimated wave vectors.

The first method (Fig. 4, panel a) is based on the estimated field-aligned current from the magnetic field measurements $\left(\delta B_{\perp}\right)$ along the satellite path $\left(\delta x=v_{s c} \delta t\right)$ through the approximate relationship

$\delta j_{\|}=\frac{\delta B_{\perp}}{\mu_{0} \lambda_{\perp}} \approx \frac{\delta B_{\perp}}{\mu_{0} \delta x}$.

The total field-aligned current fluctuations for orbit $I V$ are displayed in Fig. 5 (panel c). However, for the calculation at hand, $\delta j_{\|}$will be treated as frequency dependent through the magnetic field fluctuations. Subsequently, the parallel electric field is calculated from the polarization relation for inertial Alfvén waves (DAW with cold electrons) as

$\left|\delta E_{\|}(f)\right|=\mu_{0} 2 \pi f \lambda_{e}^{2}\left|\delta j_{\|}(f)\right|$.

The second method (Fig. 4, panel b) is calculated from the estimated perpendicular electric field $\left(\delta E_{\perp}\right)$ and the propagation angle of the DAW through

$\left|\delta E_{\|}(f)\right|=\theta \epsilon\left|\delta E_{\perp}(f)\right|$
(Seyler et al., 1995). The propagation angle of the DAW can be found from $\tan \theta=k_{\|} / k_{\perp}$, and is usually very small $\left(\theta<\epsilon=\sqrt{m_{e} / m_{i}}\right)$. When a DAW propagates at larger angles, $\theta>\epsilon$, it is known as a slow ion-acoustic (SIA) wave (Seyler and Wahlund, 1996; Seyler et al., 1998; Wahlund et al., 1998; Stasiewicz et al., 2000). The angle of propagation (Fig. 4, panel d) is small for low frequencies. Above the local gyrofrequency for $\mathrm{O}^{+}$(near $30 \mathrm{~Hz}$ on orbit $I V$ ), the propagation angle reaches $\epsilon$, consistent with the proposition that SIA emissions start to dominate the ELF emission characteristics (Wahlund et al., 1998). The $\delta E_{\perp} / \delta B_{\perp}$ ratio for a SIA wave should approach $10^{8}-10^{9} \mathrm{~m} / \mathrm{s}$, which is also consistent with the observed values presented here (Fig. 3, panel g).

The third method (Fig. 4, panel c) of estimating $\delta E_{\|}$is based on Eq. (5) above. Parallel electric field strengths of up to $0.1-1 \mathrm{mV} / \mathrm{m}$ are inferred from methods 2 and 3 (panels $b$ and c) during active times, while significantly lower values are obtained from method 1 . The increase at the upper frequencies in panel (a) is the result of the fact that the fluxgate magnetometer reaches the noise level. The orbit IV, panels (b) and (c), has the largest parallel electric field. The panels (b) and (c) are calculated using the electric and magnetic field measurements while the panel (a) is calculated from the magnetic field measurements.

\subsection{Effect on DAW induced field-aligned current systems}

The total field-aligned current variation is calculated from the fluxgate magnetometer data according to Eq. (6) and displayed for orbit $I V$ in Fig. 5 (panel c). Values reach up to $400 \mu \mathrm{A} / \mathrm{m}^{2}$. The instrument resolution gives an error of $\sim 40 \mu \mathrm{A} / \mathrm{m}^{2}$. The largest current fluctuations, which are primarily due to the ELF electromagnetic emissions, are detected when the Langmuir probe (panel a) detects plasma density depletions/cavities together with enhanced Poynting fluxes (Fig. 3, panel f).

The net integrated current along the satellite path $\left(J_{\|}\right)$is displayed in panel (b). This current gives information on where the large-scale current systems are situated. The net integrated current over a cavity is small, as can be most clearly seen near $+53 \mathrm{~s},+88 \mathrm{~s},+130 \mathrm{~s}$, and +141 s, i.e. the effect of DAW activity is small on the overall field-aligned current system. Instead, the Alfvénic activity creates a local region of field-aligned currents through the acceleration of the low energy plasma. Field-aligned electrons move downwards by the parallel electric field and transverse ion heating occurs possibly through intermediate, more electrostatic ELF waves. As a result, the plasma density drops, which causes the increased perpendicular wave vectors and the inertial and kinetic dissipative effects enhancements. A small net fieldaligned current will remain due to the accelerated plasma.

A larger integrated current can be found in the time interval of 62-82 s (17:02:20 UT-17:02:50 UT), for which only low levels of DAW activity are found; the downward proton energy flux rises to $1 \mathrm{~W} / \mathrm{m}^{2}$ (Fig. 1 , panel b; Fig. 2, panel a) and the precipitating electrons are characterized by an isotropic, smooth, inverted-V-like population (compare 


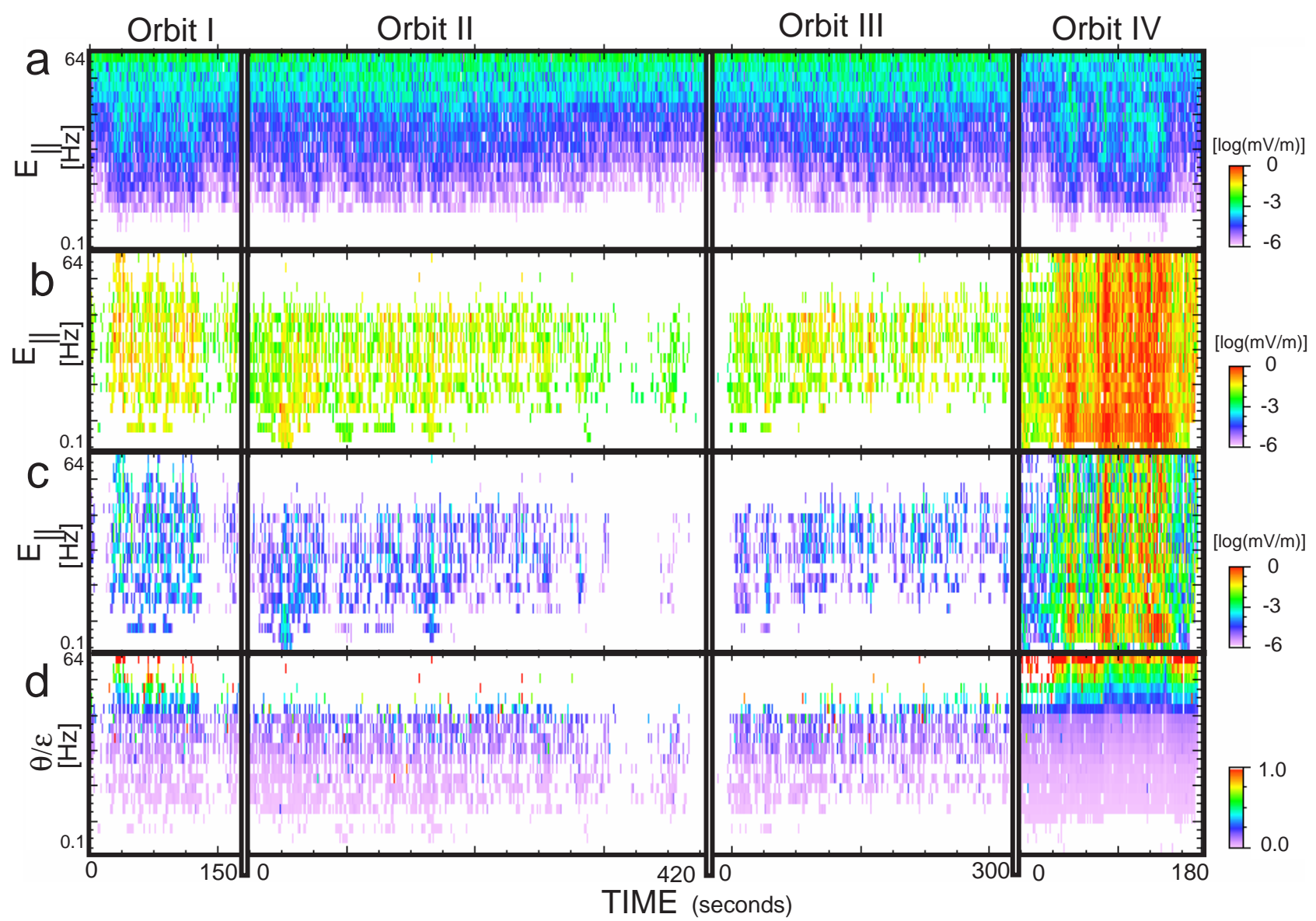

Fig. 4. The field-aligned electric field as calculated by three different methods are presented in the three top panels (see text for further details), together with the estimated propagation angle of a dispersive Alfvén wave (last panel). Parallel electric fields of the order of $0.1-1 \mathrm{mV} / \mathrm{m}$ are inferred, which can readily accelerate thermal electrons to energies of several $100 \mathrm{eV}$ within fractions of a second. Note the magnetometer noise level above $10 \mathrm{~Hz}$.

with Fig. 1, panel c, and Fig. 2, panel d). Two DAW active regions are situated at the edges of this region where the precipitating electrons probably give rise to the large integrated current by the constant flux of isotropic-with-a-losscone electrons. Since the associated field-aligned electric fields in this middle region are probably very small according to commonly used current-voltage relationships (e.g, Knight, 1973), this current, compared to that during strong Alfvén activity, is not easily cancelled out by the cold dense background plasma.

During periods when the ELF wave activity is enhanced without Langmuir probe detection of plasma density cavities, e.g. during the proton injections on orbits $I, I I$, and $I I I$ (not shown) and during the time period of 100-120s into orbit $I V$, there is also almost no net integrated current. This can be interpreted as follows. The Alfvén waves accelerate particles due to the large inertial and kinetic components and by so doing they try to create a small current. However, since this occurs in a dense background plasma, the current carried by the energetic electrons will quickly be cancelled out.

\section{Correlation study}

The electromagnetic ELF wave characteristics (wave amplitudes and $\delta E_{\perp} / \delta B_{\perp}$ ) have been correlated with various electron characteristics (energy, spectral shape, downward energy flux), as well as the plasma density for four selected spectral wave emission frequencies between $1-46 \mathrm{~Hz}$. Due to the DC-magnetometer roll off, only frequencies below $10 \mathrm{~Hz}$ can be used for the magnetic correlation. The correlations are based on 16600 points, i.e. the data presented in Fig. 3.

\subsection{Particle spectral fits}

The electron spectral shape is approximated by an exponential fit to the TESP data and characterized by the two parameters $\gamma$ and $\beta$ according to

$f_{0}(E, \phi)=\gamma(E) \cdot e^{\beta(E) \alpha}$,

where $\alpha$ represents the pitch-angle and $f_{0}$ is the measured count rate. The electron spectral fits were carried out for pitch-angles $0^{\circ}, 45^{\circ}$ and $90^{\circ}$ for which $\alpha$ was set to 0,1 and 2 , respectively, for simplicity. The parameter $\beta$ represents 


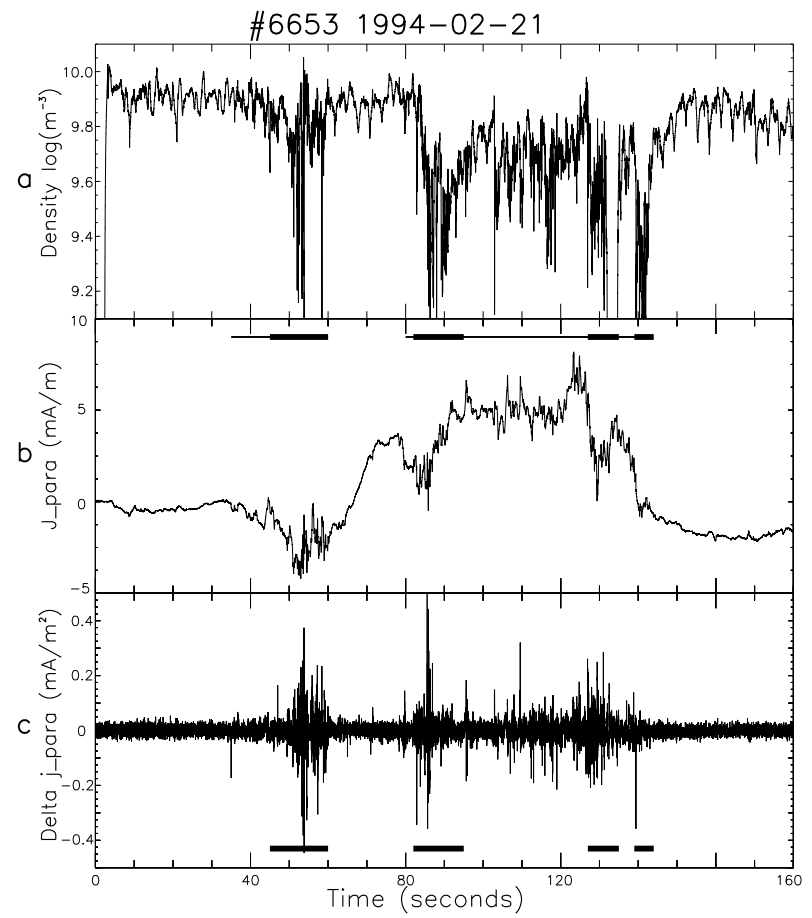

Fig. 5. The plasma density estimated from the Langmuir probe measurements (panel a) together with the estimated field-aligned current density assuming the Freja spacecraft encounters spatial structures (panel c), and the corresponding integrated net current (panel b). Alfvén wave activity results in localized regions with rather small net integrated currents on top of larger scale current systems. The thick horizontal lines in panels (b) and (c) represent where significant density drops can be found, panel (a) (note a probe sweep at +0 and +132 seconds). The thin horizontal lines, panel (b), represent where DAW is seen.

the degree to which the electron population was isotropic, $\beta \sim 0$, or field-aligned, $\beta \ll 0$ (i.e. spectral shape), and parameter $\gamma$ is the magnitude of the flux in the field-aligned direction. It is not possible to perform a lag correlation (shift in time) between the field fluctuations and various characteristics of the detected particles due to the fact that low frequencies correspond to large times, while the bursts occur during much shorter times.

\subsection{Column 1: field-aligned count dependence}

The results of the parameter fits for an electron energy of $55 \mathrm{eV}$ are compared with the electric field strength (Fig. 6, column 1). Contour lines of the constant density of the points are drawn in the figure in order to better resolve the statistical shape of the correlation. The comparison with the magnetic field strength and $\delta E_{\perp} / \delta B_{\perp}$ have also been made, but not shown.

Both the electric and magnetic field amplitudes, and consequently the $\delta E_{\perp} / \delta B_{\perp}$-ratio, correlate positively with the electron flux counts (parameter $\gamma$ ), except for the higher frequency channels of the magnetic field fluctuations.

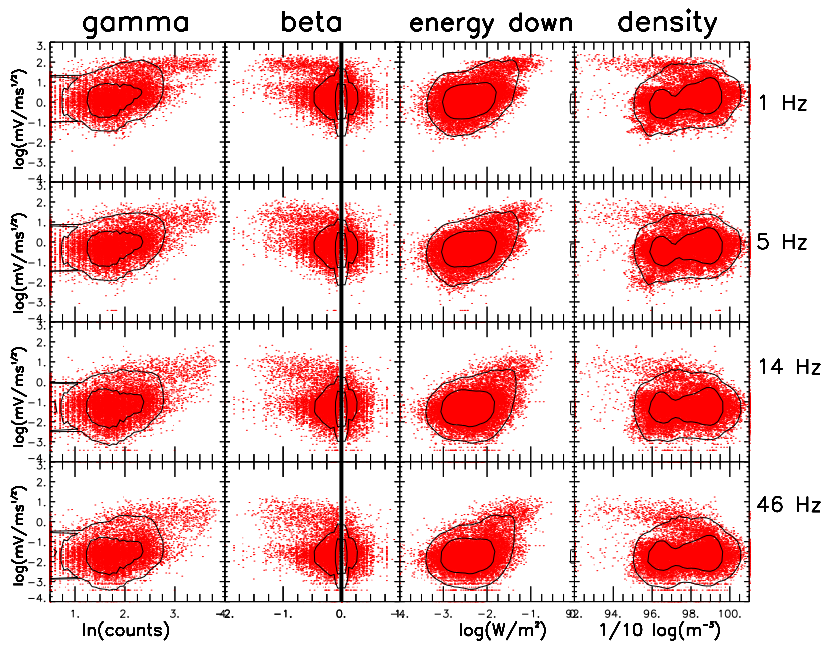

Fig. 6. Correlations between the electrons field fluctuations at 4 selected frequencies $(1,5,14$, and $46 \mathrm{~Hz})$ with particle measurements. Four types of correlations are presented; column 1 and 2, the fieldaligned electron count rate at $55 \mathrm{eV}$ and the electron spectral shape; column 3 correlates the total downward electron energy flux below $50 \mathrm{eV}$; column 4 correlates the plasma density with the field fluctuations.

\subsection{Column 2: electron spectral shape dependence}

Electron spectral shape values $(|\beta|)$ of less than 0.6 should be regarded as representing isotropic electron spectra, while $|\beta|>0.6$ indicate more field-aligned populations. The more negative the value; the more field-aligned the spectral shape, Fig. 6, column 2. The more field-aligned electron populations appear when the electric field amplitudes increase. The result is valid for all displayed frequencies.

The electron spectra at the low energy of $55 \mathrm{eV}$ show a better correlation with the amplitude of the waves compared to a higher electron energy (not shown). This is not so surprising since the low energy field-aligned electrons are suggested to be accelerated more easily by the Alfvén waves than higher energy electrons (Andersson et al., 2001). In addition, the high energy electrons, which are observed with a broad pitch-angle distribution (low value of $\beta$ ), are suggested to arrive before the Alfvén waves themselves.

\subsection{Column 3: Electron energy flux dependence}

The total downward energy flux within the energy range between $\sim 20-50 \mathrm{eV}$ is correlated with the field characteristics. The result from this correlation follows in much the same way as the correlation with the field-aligned electron count rate at $55 \mathrm{eV}$, but it is better. The contour curves indicate a good correlation between both the electric and magnetic (not shown) field amplitudes with increased downward energy flux. 


\subsection{Column 4: Plasma density dependence}

The correlations of the plasma density with the field amplitudes show that high plasma densities (i.e. outside cavities) are not well correlated or show a weak positive dependence with the electromagnetic wave fields. Within plasma cavities, on the other hand, the electric field amplitudes become large and a strong negative dependence can be found for electric field amplitudes above a threshold value. The magnetic field strength hardly correlates with the plasma density at all (not shown), indicating that the magnetic fluctuations are not important for the formation of plasma cavities and that it is the more electrostatic component of the ELF wave activity that is related to the cavity formation. The result here should be treated carefully since the density differences are less than an order of magnitude.

There are two expalnations for the correlation between the large electric field amplitude and the density cavities: the electric field empties the region of particles, thereby creating lower densities or the density cavity trapps the wave which can lead to electric field growth. In Fig. 6, column 4, the strong negative dependence might indicate that the first process is the most likely one. The good correlation between wave activity and low energy field-aligned electron precipitation, and the anti-correlation between wave activity and plasma density, has previously been reported by Chaston et al. (1999).

\section{Summary and conclusions}

In the four analyzed events, wave-particle interactions occurred together with intense downward proton injections. The correlation with dayside downward proton injection and wave-particle interactions is frequently seen in both Freja and FAST (Chaston et al., 1999) data. The ion injection can be assumed to be produced by a common process which also creates the Alfvén wave activity which, in turn, interacts with electrons through its dispersive induced field-aligned electric field in its wave front. Alternatively, an ion injection gives rise to the Alfvén wave activity which can accelerate the electrons. A scenario can be visualized in which the ratio $T_{i} / T_{e}$ plays an important role by becoming large enough so that the Alfvén wave approaches the electrostatic limit and begins to accelerate more and more electrons and at the same time, transversely heats the bulk ion population and raises the $T_{i} / T_{e}$ ratio further (Clark and Seyler, 1999). This positive feedback process then increases the effect of the inertial and kinetic terms further, and more energy dissipation can occur and so on.

The Freja mission was in operation during declining solar activity condition. The presence of Alfvén waves is identified when the $\delta E_{\perp} / \delta B_{\perp}$ ratio becomes equal to or exceeds the local Alfvén velocity. The variations in the plasma density, the Earth's magnetic field strength and the ion composition with geocentric distance cause the Alfvén speed to have a maximum below $2 R_{E}$ geocentric distance.
During orbit $I V$ (6653), which is an extreme dayside event recorded by the Freja satellite, the largest plasma temperatures were encountered and density depletions/cavities were found. In addition, during this orbit, the $\delta E_{\perp} / \delta B_{\perp}-$ ratio became significantly larger than the local Alfvén speed $\left(v_{A}\right)$ at frequencies above the local oxygen gyro frequency (about $30 \mathrm{~Hz}$ ). Furthermore, the largest perpendicular wave vector was found at these larger frequencies, and the wave characteristics became more electrostatic with increasing frequency. The data were, therefore, consistent with an interpretation in terms of dispersive Alfvén waves (Lysak and Lotko, 1996), which attain slow ion acoustic characteristics at larger frequencies (e.g. Seyler et al., 1995; Wahlund et al., 1998).

The data presented in this paper support an earlier analysis (Chaston et al., 1999) that strong ELF wave activity, especially in the electrostatic limit, is associated with larger scale plasma density depletions/cavities and at the same time, enhances the low-energy electron fluxes along the geomagnetic field lines. The correlation study presented also gives a good correlation between electromagnetic field fluctuations and the presence of accelerated electrons with energies corresponding to values below the local Alfvén velocity. Intense electrostatic ELF emissions were found to correlate well with the occurrence of density cavities.

During periods of large plasma densities, broadband ELF wave activity and bursty electrons, the integrated fieldaligned net currents were zero or very small. When larger scale density depletions/cavities were detected from the Langmuir probe measurements, the integrated field-aligned net currents became more significant, although they remained rather small. The fluctuating field-aligned current densities, however, are large, reaching several $100 \mu \mathrm{A} / \mathrm{m}^{2}$. The largest integrated field-aligned net currents were detected in association with periods of low or zero ELF wave activity. These regions have, on both sides, strong dispersive Alfvén wave activity. At those times, the fluctuations in the field-aligned current densities were, on the contrary, very low. A possible explanation is that the more isotropic electron energy distribution carries current and does not really affect the local plasma, whereas the large parallel electric fields associated with dispersive Alfvén wave activity interact heavily with the local plasma, and the local plasma, in turn, try to cancel out the fluctuating field-aligned currents created by the impulsive Alfvén waves. An impulsive Alfvén wave will effect the local plasma, causing acceleration and heating, but the total net current will be small. The flux tube associated with a large net current might be closed in the ionosphere, while the dispersive Alfvén induced currents may have a more complex fate.

Acknowledgement. The author would like to thank N. Ivchenko for his help with Freja electric and magnetic field data and the discussions. The Freja project was supported by the Swedish National Space Board (SNSB) and by the Deutsche Agentur für Raumfahrtangelegenheiten (DARA). The NSSDC OMNIWeb database was used to get information about the solar wind.

Topical Editor G. Chanteur and authors thank Y. Galperin and K. Lynch for their help in evaluating this paper. 


\section{References}

Andersson, L., Ivchenko, N., Clemmons, J., Namgaladez, A., Gustavsson, B., Wahlund, J.-E., and Eliasson, L.: Electron signatures in Alfvén waves, accepted in J. Geophys. Res., 2001.

Boehm, M. H., Carlson, C. W., McFadden, J. P., Clemmons, J. H., Mozer, F. S.: High-resolution sounding rocket observations of large-amplitude Alfvén waves, J. Geophys. Res., 95, 12 15712 171, 1990.

Boehm, M., Paschmann, G., Clemmons, J., Höfner, H., Frenzel, R., Ertl, M., Haerendel, G., Hill, P., Lauche, H., Eliasson, L., and Lundin, R.: The TESP electron spectrometer and correlator on Freja, Space Sci. Rev., 70, 3/4, 509-540, 1994.

Chaston, C. C., Carlson, C. W., Peria, W. J., Ergun, R. E., and McFadden, J. P.: FAST observations of inertial Alfvén waves in the day side aurora, Geophys. Res. Lett., 26, 647-650, 1999.

Chmyrev, V. M., Berthelier, A., Jorjio, N. V., Berthelier, J. J., Bosqued, J. M., Galperin, Yu. I., Kovrazhkin, R. A., Beghin, C., Mogilevsky, M. M., Bilichenko, S. V., and Molchanov, O.A.: Non-linear Alfvén wave generator of auroral particles and ELF/VLF waves, Planet. Space. Sci., 37, 749-759, 1989.

Clark, A. E. and Seyler, C. E.: Electron beam formation by smallscale oblique inertial Alfvén waves, J. Geophys. Res., 104, 17 233-17 249, 1999.

Eliasson, L., Norberg, O., Lundin, R., Lundin, K., Olsen, S., Borg, H., André, M., Koskinen, H., Riihelä, P., Boehm, M., and Whalen, B.: The Freja hot plasma experiment - instrument and first results, Space Sci. Rev., 70, 3/4, 563-576, 1994.

Goertz, C. K. and Boswell, R. W.: Magnetosphere-ionosphere coupling, J. Geophys. Res., 84, 7239-7246, 1979.

Goertz, C. K.: Kinetic Alfvén waves on auroral field lines, Planet. Space Sci., 32, 1387, 1984.

Haerendel, G.: An Alfvén wave model of auroral arcs, in: "HighLatitude Space Plasma Physics", (Eds) Hultqvist, B. and Hagfors, T., Plenum New York, USA, 515, 1983.

Hasegawa, A.: Particle acceleration by MHD surface wave and formation of aurora, J. Geophys. Res., 81, 5083, 1976.

Holback, B., Jansson, S.-E., Åhlén, L., Lundgren, G., Lyngdal, L., Powell, S., and Meyer, A.: The Freja wave and plasma density experiment, Space Sci. Rev., 70, 3/4, 577-592, 1994.

Ivchenko, N., Marklund, G., Lynch, K. A., Pietrowski, D., Torbert, R. B., Primdahl, F., and Ranta, A.: Quasiperiodic oscillations observed at the edge of an auroral arc by Auroral Turbulence 2, Geophys. Res. Let., 26, 3365-3368, 1999.

Knight, S.: Parallel electric fields, Plant. Space Sci., 21, 281-289, 1973.

Knudsen, D. J., Kelly, M. C., Earle, G. D., Vickrey, J. F., and Boehm, M.: Distinguishing Alfvén waves from quasistatic field structures associated with the discrete aurora: Sounding rocket and HILAT satellite measurements, Geophys. Res. Let., 921, 17, 1990.
Knudsen, D. J., Kelley, M. C., and Vockery, J. F.: Alfvén waves and the auroral ionosphere: A numerical model compared with measurements, J. Geophys. Res., 97, 77, 1992.

Knudsen, D. J. and Wahlund, J.-E.: Core ion flux bursts within solitary kinetic Alfvén waves, J. Geophys. Res., 103, 4157, 1998.

Knudsen, D. J., Clemmons, J. H., Wahlund, J.-E.: Correlation between core ion energization, suprathermal electron bursts, and broadband ELF plasma waves, J. Geophys. Res., 103, 41714186, 1998.

Louarn, P., Wahlund, J.-E., Chust, T., de Feraudy, H., Roux, A., Holback, B., Dovner, P. O., Eriksson, A. I., and Holmgren, G.: Observations of kinetic Alfvén waves by FREJA spacecraft, Geophys. Res. Lett., 21, 1847, 1994.

Lynch, K. A., Pietrowski, D., Torbert, R. B., Ivchenko, N., Marklund, G., Primdahl, F.: Multiple-point electron measurements in a nightside auroral arc: Auroral Turbulence II particle observations, Geophys. Res. Let., 26, 3361-3364, 1999.

Lysak, R. L. and Lotko, W.: On the kinetic dispersion relation for shear Alfvén waves, J. Geophys. Res., 101, 5085-5094, 1996.

Marklund, G. T., Blomberg, L. G., Lindqvist, P.-A., Fälthammar, C.-G., Haerendel, G., Mozer, F., Pedersen, A., and Tanskanen, P.: The double probe electric field experiment on Freja: Expriment description and first results, Space Sci. Rev., 70, 3/4, 483-508, 1994.

Seyler, C. E., Wahlund, J.-E.: Theory of nearly perpendicular electrostatic plasma waves and comparison to Freja satellite observations, J. Geophys. Res., 101, 21 795-21 813, 1996.

Seyler, C. E., Wahlund, J.-E., and Holback, B.: Theory and simulation of low-frequency plasma waves and comparison to Freja satellite observations, J. Geophys. Res., 100, 21 453-21472, 1995.

Seyler, C. E., Clark, A. E., Bonnell, J., and Wahlund, J.-E.: Electrostatic broadband ELF wave emission by Alfvén wave breaking, J. Geophys. Res., 103, 7027-7041, 1998.

Stasiewicz, K., Bellan, P., Chaston, C., Kletzing, C., Lysak, R., Maggs, J., Pokhotelov, O., Seyler, C. E., Shukla, P., Stenflo, L., Stretsov, A., and Wahlund, J.-E.: Small scale Alfvénic structure in the aurora, Space Sci. Rev., 92, 423-533, 2000.

Stefant, R. J.: Alfvén wave damping from finite gyroradius coupling to the ion acoustic mode, Phys. Fluids., 13, 440, 1970.

Wahlund, J.-E., Louarn, P., Chust, T., de Feraudy, H., Roux, A., Holback, B., Cabrit, B., Eriksson, A. I., Kintner, P. M., Kelley, M. C., Bonnell, J., and Chesney, S.: Observation of ion acoustic fluctuations in the auroral topside ionosphere by the FREJA s/c, Geophys. Res. Let., 21, 1835-1838, 1994.

Wahlund, J.-E., Ericsson, A. I., Holback, B., Boehm, M., Bonnell, J., Kintner, P. M., Seyler, C. E., Clemmons, J. H., Eliasson, L., Knudsen, D. J., Norqvist, P., and Zanetti, L. J.: Broadband ELF plasma emission during auroral energization 1. Slow ion acoustic waves, J. Geophys. Res., 103, 4343-4375, 1998. 